\title{
Corneal thickness, epithelial thickness and axial length differences in normal and high myopia
} Xiaogang $W^{1+}{ }^{1 \dagger}$, Jing Dong ${ }^{2+}$ and Qiang $W^{3^{*}}$

\begin{abstract}
Background: Corneal biometric parameters can possibly be influenced by high myopia (HM). The influence of HM on corneal thickness (CT), epithelial thickness (ET) has not yet been clearly established. The aim of this study is to observe ET, CT and axial length (AL) differences between in normal and subjects with HMs and to investigate factors influencing the corneal biometric parameters and $\mathrm{AL}$, such as age and gender.

Methods: A total of 97 normal subjects (97 eyes) and $48 \mathrm{HM}$ subjects (48 eyes) were included. The ET and CT of the central 6-mm diameter (17 regions) and the AL data were captured. The 17 corneal and epithelial regions were the center ( $1 \mathrm{~mm}$ radius, area a), the inner ring $(2.5 \mathrm{~mm}$ radius, area b), the outer ring $(3 \mathrm{~mm}$ radius, area $\mathrm{c})$ and the 8 radial scan lines in eight directions (Superior (1) , SN (2), Nasal (3), IN (4), Inferior (5), IT (6), Temporal (7), ST (8)) with an angle of $45^{\circ}$ between each consecutive scan line $(a, b$ 1-8, c 1-8).

Results: The ALs were increased about $4 \mathrm{~mm}$ in the HMs $(P<0.001)$. No differences in ET were observed; in contrast, significantly thicker CTs were observed in the HMs in 16 regions except the b5 subregion. In normal group, age was negatively correlated with AL but not CCT and CET and gender was correlated with CET. In HM group, age was not correlated with CCT, AL or CET and gender was correlated with AL and CCT but not CET.
\end{abstract}

Conclusions: $C T$ was thicker in the HMs but not ET. Age and gender should be considered for AL, CT and ET in both normal and HM group.

Keywords: Axial length, Corneal thickness, Epithelial thickness, High myopia, Optical coherence tomography

\section{Background}

Corneal biometric parameters and axial length (AL) can possibly be influenced by the degrees of refractive error. The influence of refractive error on corneal thickness (CT), epithelial thickness (ET) has not yet been clearly established [1-3]. High myopia (HM) plays an important role in visual impairment and is highly common in China, particularly in the school population $[4,5]$. HM patients are at greater risks of posterior subcapsular cataracts, glaucoma and chorioretinal abnormalities [6]. The known severe visual problems primarily result from posterior segment complications, such as macular holes, choroidal neovascularization, retinal detachment, retinoschisis, etc. Regarding the anterior segment, HM may affect $\mathrm{CT}$ in different age groups $[1,2]$. However, little

\footnotetext{
* Correspondence: movie6521@gmail.com

'Equal contributors

${ }^{3}$ Affiliated Sixth People's Hospital Shanghai Jiao Tong University, Shanghai,

P.R. China

Full list of author information is available at the end of the article
}

research into comparing mid-peripheral $\mathrm{CT}$ and ET between normal subjects and those with HM on Chinese populations. Moreover, the central CT (CCT) results in $\mathrm{HM}$ is controversial and this require further study to clarify the topic $[1,2,7]$.

Therefore, the purpose of this study was to evaluate CT, ET and AL differences between Chinese normal and HM groups to clarify the changing tendency of central, mid-peripheral CT, ET in Chinese HMs.

\section{Methods}

This study was performed at the Affiliated Sixth People's Hospital Shanghai Jiao Tong University (Shanghai, China). The research protocols were approved by the institutional review boards of the Affiliated Sixth People's Hospital Shanghai Jiao Tong University (Shanghai, China) and performed in accordance with the tenets of the Declaration of Helsinki. Written informed consent was obtained from each subject after they were provided with an explanation of the nature of the study. 


\section{Subjects}

We just chose Han Chinese subjects to eliminate the possible influences of different ethnic groups. The normal and HM subjects were chosen from the Ophthalmic Clinic Center at the Shanghai Sixth People's Hospital. One random eye of each subject was chosen for this study. The inclusion criteria for the normal subjects included the following: a best-corrected visual acuity (BCVA) of $\geq 16 / 20$, a refractive error $<5$ diopter (D) spheres, normal slit-lamp and fundoscopy examinations, an IOP $<22 \mathrm{mmHg}$, and no history of ocular or systemic corticosteroid use. The inclusion criteria for the HM subjects were as follows: a BCVA of $\geq 20 / 40$, a spherical refractive error more negative than -6 diopters, and central fixation sufficiently stable to perform image capture. Subjects with keratoconus, previous corneal lesions and prior surgery in the cornea, severe cataracts, glaucoma or posterior abnormalities, such as choroidal neovascularization, retinoschisis, retinal detachment or macular holes, were excluded.

\section{ET and CT measurement}

An Optovue RTVue (Optovue Inc., Fremont, CA, USA) and a supplemental cornea-anterior module (CAM) attachment were used for the epithelial and corneal pachymetry scans. Several studies have shown that RTVue has excellent repeatability and reproducibility in both CT and ET measurements $[8,9]$. The RTVue scans an area of $6 \times 6 \mathrm{~mm}$ in the central cornea with a depth resolution approximately $5 \mu \mathrm{m}$. The light source of the system uses super luminescent diodes with wavelengths of $840 \mathrm{~nm}$. Eight radial line scans with 1024 A-scans each for the corneal and epithelial pachymetry maps are used to demonstrate the average CT and ET in each of the 17 regions as described in our previous research [10]. These regions (Figure 1) are the center (1 mm radius, area a), the inner ring $(2.5 \mathrm{~mm}$ radius, area $\mathrm{b})$, the outer ring ( $3 \mathrm{~mm}$ radius, area $\mathrm{c}$ ), and the eight radial scan lines in eight directions (superior (1), SN (2), nasal (3), IN (4), inferior (5), IT (6), temporal (7), and ST (8)) with angles of $45^{\circ}$ between each consecutive scan line (a, b 1-8, c 1-8)). All of the images in this study were captured by an experienced and trained technician. All of the corneal and epithelial pachymetry data were automatically calculated by the RTVue software (version 6.11.0.12). During each scan, the technician captured each cross-sectional corneal image with the light beam at the midpoint to ensure that the scan location was in the center area of the cornea.

\section{AL measurement}

A Lenstar LS 900 biometer (Haag-Streit AG, Koeniz, Switzerland) and its internal software (version 2.1.1)were used for the AL measurements. The Lenstar LS 900 biometer uses optical low-coherence reflectometry and a central wavelength of $820 \mu \mathrm{m}$ to measure the AL to the

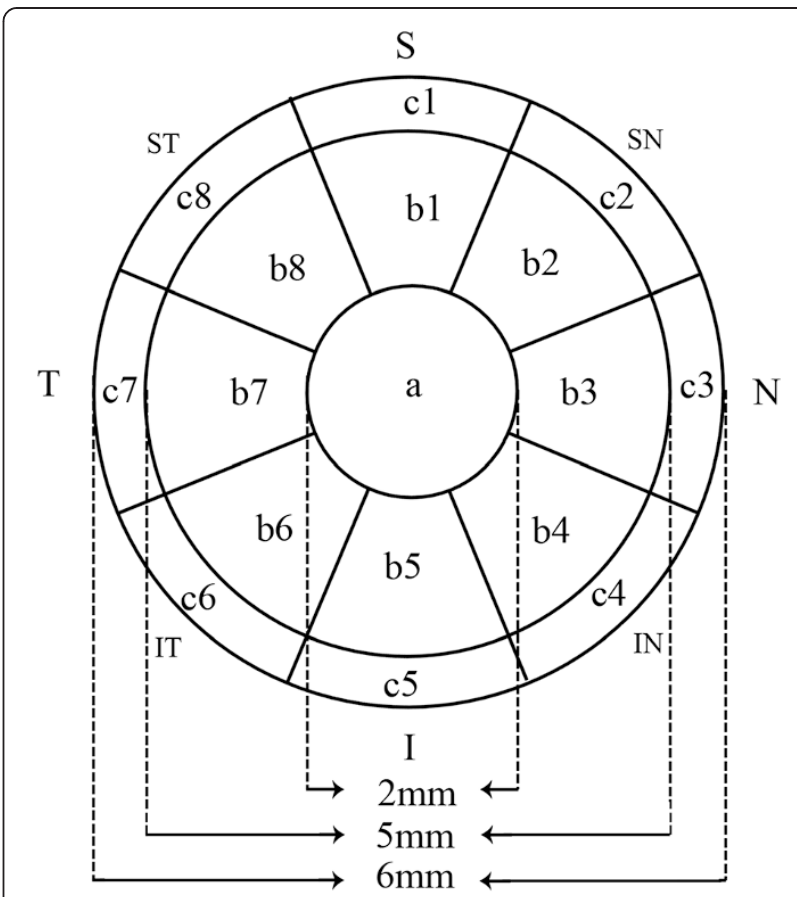

Figure 1 Pachymetry map of the corneal and epithelial thicknesses of 17 regions of the central $6 \mathrm{~mm}$. Temporal (T); nasal $(\mathrm{N})$; superior (S); inferior (I).

retinal pigment epithelium [11]. During the examination, the patients were asked to fixate on the internal red light, and the device focus was based on the image of the eye on the monitor. The patients were asked to perform a complete blink to make ensure an optically smooth tear film over the cornea prior to image capture. Measurements that were contaminated by patient blinking or unstable fixation were excluded, and one noncontaminated measurement for each eye was used in the final analysis.

\section{Statistical analyses}

The statistical analyses were performed with commercial software (SPSS ver. 13.0; SPSS Inc.). The means \pm the standard deviations of each variable were assessed for both the normal and HM groups. To compare the ET, CT and $\mathrm{AL}$ variables measured in the normal and HM eyes, independent sample t-tests were used. The correlation coefficients were also calculated for age, gender, ET, CT and AL in each group. The significance level for all of the tests was set at $5 \%$.

\section{Results}

Data from one random eye of 97 normal subjects and 48 HM patients were analyzed (Table 1 ). There was no significant difference in age between the 2 groups. However, there were significant difference in gender, spherical error and astigmatism between the 2 groups. 
Table 1 Characteristics of normal subjects and subjects with high myopia

\begin{tabular}{llll}
\hline Characteristics & Normal & High myopia & $\boldsymbol{P}$ Value* \\
\hline Patients, $\mathrm{n}$ & 97 & 48 & \\
Eyes, $\mathrm{n}$ & 97 & 48 & \\
Age (yrs) & $56 \pm 18$ & $51 \pm 20$ & 0.188 \\
Gender (male/female) & $33 / 64$ & $25 / 23$ & $\mathbf{0 . 0 3 7}$ \\
Spherical error (diopter) & $-1.27 \pm 0.64$ & $-9.11 \pm 3.63$ & 0.000 \\
Astigmatism (diopter) & $0.89 \pm 0.50$ & $1.45 \pm 0.89$ & 0.000 \\
\hline
\end{tabular}

*All calculated by $\mathrm{t}$ test, except the values in bold, which was calculated by chi-square test.

Significant differences in the CT and AL values were found between the normal and HM eyes, but no difference was found in the ET values (Table 2, Figure 2). Compared to the normal eyes, the ALs of the HM eyes were significantly increased by $16 \%(\mathrm{P}<0.001)$. In contrast to our finding of no difference in the ETs, significantly thicker CTs in the HM eyes were found in all 17 regions, and the $\mathrm{c} 1, \mathrm{c} 2$ and $\mathrm{c} 8$ regions exhibited the largest biggest differences.

Age was negatively associated with AL but not CCT and $\mathrm{CET}$ in normal group. In the HM group, age was not associated with CCT , AL or CET. In the normal group, gender has significant correlation with CET but no CCT and $\mathrm{AL}$; in the HM group, gender was correlated with CCT and AL but not with CET (Table 3).

\section{Discussion}

Due to the longer eyeballs of the HM subjects, both the anterior and posterior segments exhibited some abnormalities $[6,12]$. In this study, we evaluated the correlations between age, gender, CT, ET and AL in normal and HM groups. We also investigated the differences in the CTs, ETs, ALs between the two groups. Similar to the scatter plot showing a strong association between higher myopia and longer ALs shown in the study by Kubo et al., significantly longer ALs were found in the HM group than in the normal group in the present study [13].

As the first cellular layer of the human cornea, the corneal epithelium plays an important role in the evaluation of corneal remodeling after refractive surgery [14]. Several technologies can measure ET, such as confocal microscopy, very-high frequency ultrasound, time-domain optical coherence tomography (TD-OCT) and spectral-domain optical coherence tomography (SD-OCT) [15-18]. Reinstein et al. reported CET of $53.4 \pm 4.6 \mu \mathrm{m}$ in normal corneas using very-high frequency ultrasound [16]. The measurements of Reinstein et al., which did not include precorneal tear film thickness, were thinner than the CET of $56 \pm 3.6 \mu \mathrm{m}$ of the normal eyes found in the present study. Tear film thickness measurements were included in
Table 2 Summary of the axial length, corneal thickness and epithelial thickness of normal and high myopia groups

\begin{tabular}{|c|c|c|c|c|}
\hline & $\begin{array}{l}\text { Normal } \\
(n=97)\end{array}$ & $\begin{array}{l}\mathrm{HM} \\
(\mathrm{n}=48)\end{array}$ & $\begin{array}{l}\text { Mean } \\
\text { difference }\end{array}$ & $P$ \\
\hline Axial length (mm) & $23.1 \pm 0.7$ & $26.7 \pm 2.1$ & -3.6 & 0.000 \\
\hline \multicolumn{5}{|l|}{$C T(\mu \mathrm{m})$} \\
\hline Central (a) & $526 \pm 29$ & $539 \pm 34$ & -14 & 0.013 \\
\hline$S(b 1)$ & $559 \pm 34$ & $575 \pm 37$ & -16 & 0.011 \\
\hline $\mathrm{SN}(\mathrm{b} 2)$ & $551 \pm 33$ & $567 \pm 37$ & -16 & 0.009 \\
\hline N (b3) & $540 \pm 31$ & $555 \pm 35$ & -14 & 0.014 \\
\hline IN (b4) & $538 \pm 30$ & $550 \pm 33$ & -12 & 0.030 \\
\hline I (b5) & $540 \pm 31$ & $550 \pm 31$ & -11 & 0.054 \\
\hline IT (b6) & $538 \pm 31$ & $549 \pm 32$ & -11 & 0.041 \\
\hline $\mathrm{T}(\mathrm{b} 7)$ & $541 \pm 32$ & $555 \pm 34$ & -13 & 0.023 \\
\hline ST (b8) & $553 \pm 33$ & $568 \pm 35$ & -15 & 0.013 \\
\hline$S(c 1)$ & $590 \pm 41$ & $608 \pm 41$ & -18 & 0.014 \\
\hline $\mathrm{SN}(\mathrm{c} 2)$ & $572 \pm 37$ & $590 \pm 40$ & -18 & 0.008 \\
\hline$N(c 3)$ & $558 \pm 33$ & $573 \pm 36$ & -15 & 0.016 \\
\hline IN (c4) & $558 \pm 32$ & $571 \pm 33$ & -13 & 0.023 \\
\hline I (c5) & $562 \pm 33$ & $574 \pm 30$ & -12 & 0.041 \\
\hline IT (c6) & $556 \pm 33$ & $567 \pm 30$ & -11 & 0.046 \\
\hline $\mathrm{T}(\mathrm{c} 7)$ & $561 \pm 35$ & $574 \pm 34$ & -13 & 0.034 \\
\hline ST (c8) & $578 \pm 36$ & $596 \pm 38$ & -18 & 0.008 \\
\hline
\end{tabular}

ET $(\mu \mathrm{m})$

Central (a)

\begin{tabular}{llll}
$56 \pm 3.6$ & $55 \pm 2.9$ & 0.7 & 0.214 \\
$53 \pm 4.3$ & $53 \pm 3.1$ & 0.3 & 0.677 \\
$53 \pm 4.1$ & $53 \pm 3.1$ & 0.1 & 0.870 \\
$54 \pm 3.8$ & $54 \pm 2.7$ & -0.2 & 0.763 \\
$55 \pm 3.8$ & $55 \pm 2.5$ & 0.1 & 0.803 \\
$56 \pm 3.7$ & $55 \pm 2.6$ & -0.004 & 0.994 \\
$55 \pm 3.6$ & $55 \pm 2.6$ & 0.1 & 0.891 \\
$55 \pm 3.6$ & $54 \pm 2.7$ & 0.2 & 0.746 \\
$53 \pm 3.9$ & $53 \pm 3.0$ & 0.3 & 0.656 \\
$51 \pm 4.6$ & $51 \pm 3.8$ & -0.8 & 0.327 \\
$52 \pm 4.3$ & $52 \pm 3.4$ & -1.0 & 0.154 \\
$53 \pm 4.0$ & $53 \pm 2.9$ & -0.3 & 0.571 \\
$54 \pm 4.1$ & $54 \pm 2.9$ & -0.5 & 0.437 \\
$54 \pm 4.3$ & $55 \pm 2.9$ & -0.8 & 0.182 \\
$54 \pm 4.0$ & $54 \pm 2.6$ & -0.3 & 0.588 \\
$53 \pm 3.8$ & $53 \pm 3.0$ & -0.2 & 0.754 \\
$52 \pm 4.3$ & $52 \pm 3.5$ & -0.4 & 0.614 \\
\hline
\end{tabular}

$\mathrm{CT}=$ corneal thickness; $\mathrm{ET}=$ epithelial thickness; $\mathrm{HM}=$ high myopia; I = inferior; $\mathrm{N}=$ nasal; $\mathrm{S}=$ superior; $\mathrm{T}=$ temporal.

Statistically significant differences $(P<0.05)$ are bold faced.

our study, and may account for this difference. Previous studies have reported varying CET values for normal corneas using TD/SD-OCT, even when the same brand of 

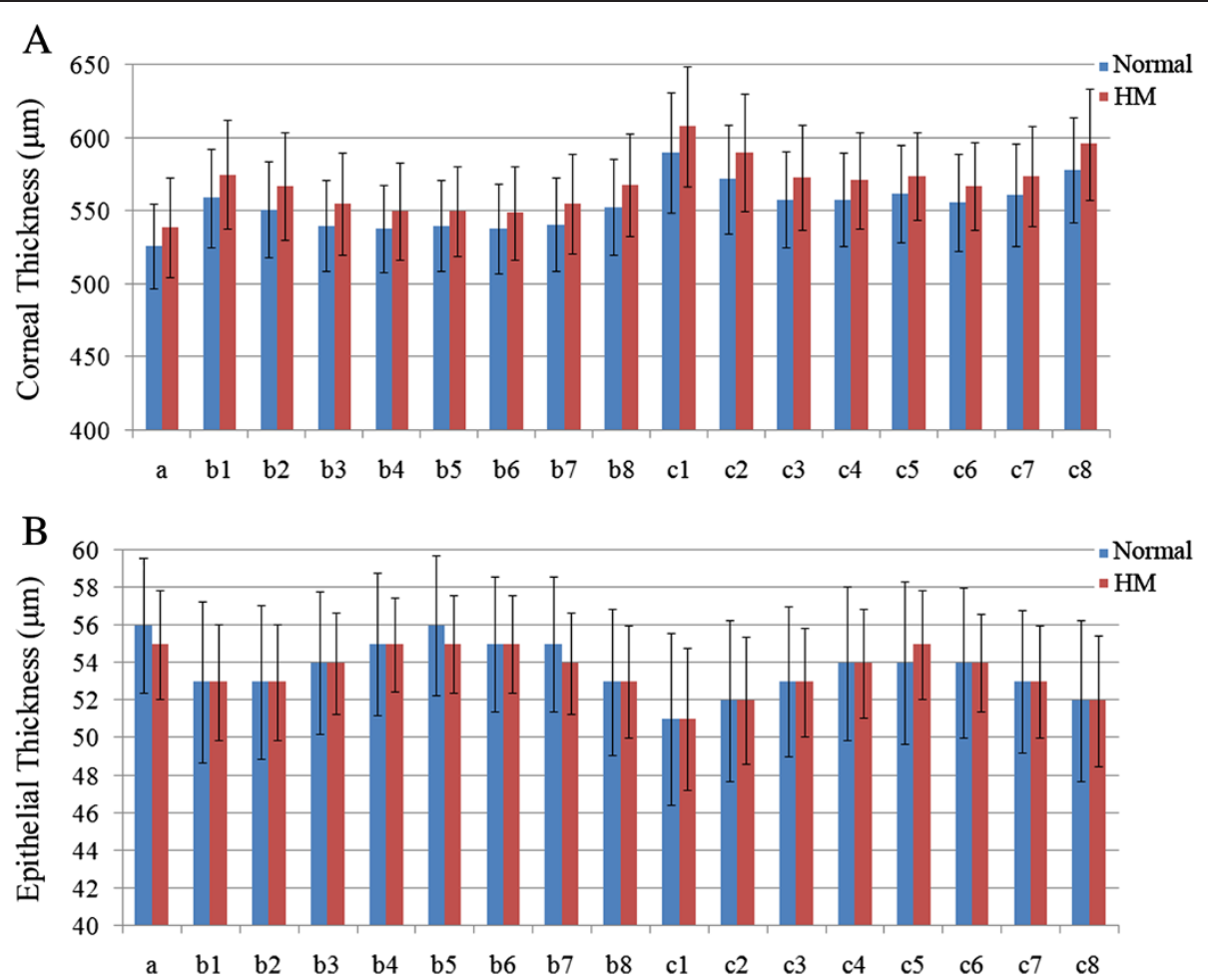

Figure $\mathbf{2}$ Histograms comparing the corneal thickness (A) and epithelial thicknesses (B) of the 17 regions between the normal and high myopia groups.

OCT device was used [17-19]. These fluctuations may be attributable to different races, ages and sample sizes.

Regarding the CCT measurements, our results are very similar to those of Wong et al. who examined a sample of normal Hong Kong Chinese people using SDOCT [20]. Compared to our previous research that has used the Galilei Scheimpflug system, OCT produced thinner CCT values $[21,22]$. This difference may be attributable to differences in the measuring techniques.

This study revealed significantly thicker CTs in 16 regions of the central $6 \mathrm{~mm}$ in the HM group. This result is in agreement with that of Kunert et al. who studied an Indian population [7]. However, this result is different from the thinner or unaltered CTs that have been reported for HM samples in previous studies [2,23]. There are several possible reasons for this difference. First, we assume that there are different stages of the changes of CT in HM; e.g., early, middle, and advanced stages. There might be different CTs associated with each stage, and research into these myopic stages should be performed. Second, CT exhibits a 24-hour fluctuation, which might have influenced the final measurement values for the statistical analyses of different studies due to differences in measurement times [24]. Third, the refractive range for $\mathrm{HM}$, which may influence the $\mathrm{CT}$, were different in each study. In contrast to the thicker CTs of the HM eyes, no differences in ET between the two groups were identified in any of the 17 sub-regions, which indicates that the changes in CT were primarily attributable to the corneal stroma.

In the present study, age was negatively associated with $\mathrm{AL}$ in the normal group, and this finding is similar

Table 3 Correlation coefficient matrix for the associations of gender, age with corneal thickness, epithelial thickness, and axial length in normal and high myopia groups

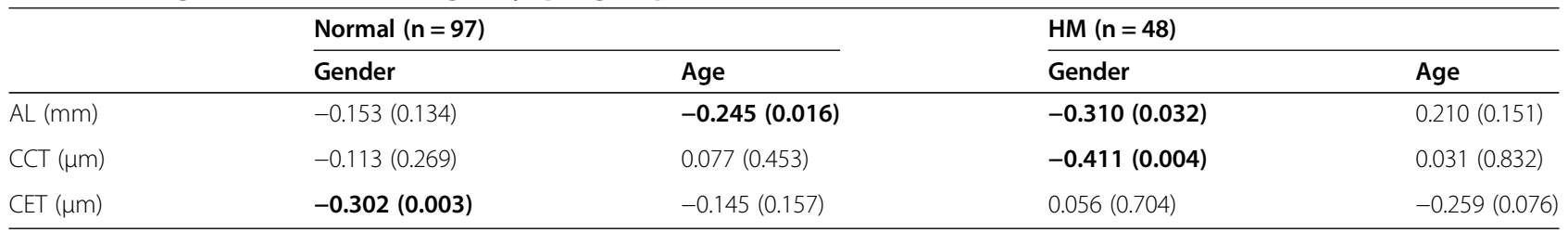

The table cells display the correlation coefficients (P-values are based on Pearson correlation analyses).

$\mathrm{CCT}=$ central corneal thickness; $\mathrm{CET}=$ central epithelial thickness; $\mathrm{HM}=$ high myopia; $\mathrm{I}=$ inferior; $\mathrm{N}=$ nasal; $\mathrm{S}=$ superior; $\mathrm{T}=$ temporal .

Statistically significant differences $(P<0.05)$ are bold faced. 
to the finding of an increase in AL with decreasing age at the time of cataract surgery of the research of Tuft et al. [25]. In contrast to AL, CCT was found to remain constant with age at the resolution available using the SD-OCT in both the normal and HM groups, which exhibited results that were similar to those of previous studies that have used the Scheimpflug system and TDOCT $[22,26]$. The influence of gender on CET in normal group and CCT, AL in HM group may attribute to the endocrine differences between man and women. Previous research revealed that gonadal hormones may affect ocular tissue growth by genomic or nongenomic pathway $[27,28]$.

The current study has some limitations. First, the normal participants were not gender matched to the HM group. Second, the potential for segmentation errors by the automated software, the drift of the measurements that can be caused by instrument vibration during operation and the signal instability should be considered. Third, additional studies with larger HM samples that include multiple races and more even gender distributions are also needed. However, The corneal biometric parameters changing in $\mathrm{HM}$ of this study may be an important indicator for refractive surgery choosing or designing.

\section{Conclusions}

In this report, the CTs of the HM group were significantly thicker, particularly in the $\mathrm{c} 1, \mathrm{c} 2$ and $\mathrm{c} 8$ subregions. Age and gender should be noticed for AL, CT and ET in both normal and HM group.

\section{Competing interests}

The authors declare that they have no competing interests.

\section{Authors' contributions}

XGW carried out this research, collected data, made analysis and interpretation of data and drafted the manuscript. JD collected data, made analysis and interpretation of data. XGW and QW conceived of the study, and participated in its design and coordination and helped to draft the manuscript. All authors read and approved the final manuscript.

\section{Author details}

${ }^{1}$ Shanxi Eye Hospital, Shanxi, P.R. China. ${ }^{2}$ The First Hospital of Shanxi Medical University, Shanxi, P.R. China. ${ }^{3}$ Affiliated Sixth People's Hospital Shanghai Jiao Tong University, Shanghai, P.R. China.

Received: 10 January 2015 Accepted: 29 April 2015

Published online: 07 May 2015

\section{References}

1. Chang SW, Tsai IL, Hu FR, Lin LL, Shih YF. The cornea in young myopic adults. Br J Ophthalmol. 2001;85(8):916-20.

2. Pedersen L, Hjortdal J, Ehlers N. Central corneal thickness in high myopia. Acta Ophthalmol Scand. 2005;83(5):539-42.

3. Al-Mezaine HS, Al-Obeidan S, Kangave D, Sadaawy A, Wehaib TA, Al-Amro SA. The relationship between central corneal thickness and degree of myopia among Saudi adults. Int Ophthalmol. 2009;29(5):373-8.

4. Sun J, Zhou J, Zhao P, Lian J, Zhu H, Zhou Y, et al. High prevalence of myopia and high myopia in 5060 Chinese university students in Shanghai. Invest Ophthalmol Vis Sci. 2012;53(12):7504-9.

5. Morgan IG, Ohno-Matsui K, Saw SM. Myopia. Lancet. 2012;379(9827):1739-48.
6. Saw SM, Gazzard G, Shih-Yen EC, Chua WH. Myopia and associated pathological complications. Ophthalmic Physiol Opt. 2005;25(5):381-91.

7. Kunert KS, Bhartiya P, Tandon R, Dada T, Christian H, Vajpayee RB. Central corneal thickness in Indian patients undergoing LASIK for myopia. J Refract Surg. 2003;19:0378-9.

8. Ma XJ, Wang L, Koch DD. Repeatability of corneal epithelial thickness measurements using Fourier-domain optical coherence tomography in normal and post-LASIK eyes. Cornea. 2013;32(12):1544-8.

9. Chen S, Huang J, Wen D, Chen W, Huang D, Wang Q. Measurement of central corneal thickness by high-resolution Scheimpflug imaging, Fourier-domain optical coherence tomography and ultrasound pachymetry. Acta Ophthalmol. 2012;90(5):449-55.

10. Wang X, Wu Q. Normal corneal thickness measurements in pigmented rabbits using spectral-domain anterior segment optical coherence tomography. Vet Ophthalmol. 2013;16(2):130-4.

11. Rohrer K, Frueh BE, Walti R, Clemetson IA, Tappeiner C, Goldblum D. Comparison and evaluation of ocular biometry using a new noncontact optical low-coherence reflectometer. Ophthalmology. 2009;116(11):2087-92.

12. Jones $\mathrm{D}$, Luensmann $\mathrm{D}$. The prevalence and impact of high myopia. Eye Contact Lens. 2012;38(3):188-96.

13. Kubo E, Kumamoto Y, Tsuzuki S, Akagi Y. Axial length, myopia, and the severity of lens opacity at the time of cataract surgery. Arch Ophthalmol. 2006;124(11):1586-90.

14. Huang D, Tang M, Shekhar R. Mathematical model of corneal surface smoothing after laser refractive surgery. Am J Ophthalmol. 2003;135(3):267-78.

15. Erie JC, Patel SV, McLaren JW, Ramirez M, Hodge DO, Maguire LJ, et al. Effect of myopic laser in situ keratomileusis on epithelial and stromal thickness: a confocal microscopy study. Ophthalmology. 2002;109(8):1447-52.

16. Reinstein DZ, Archer TJ, Gobbe M, Silverman RH, Coleman DJ. Epithelial thickness in the normal cornea: three-dimensional display with Artemis very high-frequency digital ultrasound. J Refract Surg. 2008;24(6):571-81.

17. Sin S, Simpson TL. The repeatability of corneal and corneal epithelial thickness measurements using optical coherence tomography. Optom Vis Sci. 2006;83(6):360-5.

18. Kanellopoulos AJ, Asimellis G. In vivo 3-dimensional corneal epithelial thickness mapping as an indicator of dry eye: preliminary clinical assessment. Am J Ophthalmol. 2014;157(1):63-8. e62

19. Wu S, Tao A, Jiang H, Xu Z, Perez V, Wang J. Vertical and horizontal corneal epithelial thickness profile using ultra-high resolution and long scan depth optical coherence tomography. PLoS One. 2014;9(5):e97962.

20. Wong AC, Wong CC, Yuen NS, Hui SP. Correlational study of central corneal thickness measurements on Hong Kong Chinese using optical coherence tomography, Orbscan and ultrasound pachymetry. Eye (Lond). 2002;16(6):715-21.

21. Wang X, Dong J, Wu Q. Evaluation of anterior segment parameters and possible influencing factors in normal subjects using a dual scheimpflug analyzer. PLoS One. 2014;9(5):e97913.

22. Wang $X$, Wu Q. Investigation of the human anterior segment in normal Chinese subjects using a dual Scheimpflug analyzer. Ophthalmology. 2013;120(4):703-8

23. Ucakhan OO, Gesoglu P, Ozkan M, Kanpolat A. Corneal elevation and thickness in relation to the refractive status measured with the Pentacam Scheimpflug system. J Cataract Refract Surg. 2008;34(11):1900-5.

24. Fogagnolo P, Capizzi F, Orzalesi N, Figus M, Ferreras A, Rossetti L. Can mean central corneal thickness and its 24-hour fluctuation influence fluctuation of intraocular pressure? J Glaucoma. 2010;19(6):418-23.

25. Tuft SJ, Bunce C. Axial length and age at cataract surgery. J Cataract Refract Surg. 2004;30(5):1045-8.

26. Germundsson J, Karanis G, Fagerholm P, Lagali N. Age-related thinning of Bowman's layer in the human cornea in vivo. Invest Ophthalmol Vis Sci. 2013:54(9):6143-9.

27. Giuffre G, Di Rosa L, Fiorino F, Bubella DM, Lodato G. Variations in central corneal thickness during the menstrual cycle in women. Cornea. 2007:26(2):144-6.

28. Gupta PD, Johar Sr K, Nagpal K, Vasavada AR. Sex hormone receptors in the human eye. Surv Ophthalmol. 2005;50(3):274-84. 